\title{
The ITER bolometer diagnostic: Status and plans ${ }^{a)}$
}

\author{
H. Meister, ${ }^{1, b)}$ L. Giannone, ${ }^{1}$ L. D. Horton, ${ }^{1}$ G. Raupp, ${ }^{1}$ W. Zeidner, ${ }^{1}$ G. Grunda, ${ }^{2}$ \\ S. Kalvin, ${ }^{2}$ U. Fischer, ${ }^{3}$ A. Serikov, ${ }^{3}$ S. Stickel, ${ }^{3}$ and R. Reichle ${ }^{4}$ \\ ${ }^{1}$ Max-Planck-Institut für Plasmaphysik, EURATOM Association, Boltzmannstr. 2, D-85748 Garching, \\ Germany \\ ${ }^{2}$ KFKI-Research Institute for Particle and Nuclear Physics, EURATOM Association, P.O. Box 49, \\ Budapest-114, H-1525, Hungary \\ ${ }^{3}$ Forschungszentrum Karlsruhe, EURATOM Association, Institut für Reaktorsicherheit, Postfach 3640, 76021 \\ Karlsruhe, Germany \\ ${ }^{4}$ CEA Cadarache, EURATOM Association, 13108 Saint-Paul-Lez-Durance, France
}

(Presented 15 May 2008; received 11 May 2008; accepted 6 July 2008; published online 31 October 2008)

\begin{abstract}
A consortium consisting of four EURATOM Associations has been set up to develop the project plan for the full development of the ITER bolometer diagnostic and to continue urgent R\&D activities. An overview of the current status is given, including detector development, line-of-sight optimization, performance analysis as well as the design of the diagnostic components and their integration in ITER. This is complemented by the presentation of plans for future activities required to successfully implement the bolometer diagnostic, ranging from the detector development over diagnostic design and prototype testing to RH tools for calibration. (C) 2008 American Institute of Physics. [DOI: 10.1063/1.2965002]
\end{abstract}

\section{INTRODUCTION}

As the total radiated power from the plasma enters the overall energy balance, it is a crucial parameter to be determined for the successful operation of any fusion device. The total radiation as well as the radiation emission profile can be determined by the bolometer diagnostic. The bolometer diagnostic of ITER is contained in the procurement package 21 (PP21) which was allocated to the EU. In 2006 a consortium of five EURATOM Fusion Associations was set up under the lead of the Max-Planck-Institute for Plasmaphysics (IPP) to prepare the project plan for the full development of PP21 and to continue with the most urgent $R \& D$ within the framework of a task of the EFDA Technology Workprogramme. As the leading association, IPP provided the project lead, oversaw the detector development, and contributed to the engineering activities. The Hungarian Association continued with their efforts on the line-of-sight (LOS) optimization and performance analysis, performed the thermal analysis, and contributed to the engineering activities. FZK performed the nuclear analysis and the French Association assessed the status of alternative detector types for ITER (imaging and capacitive bolometers) and contributed to the irradiation tests. The Spanish Association worked as an independent subproject on the pressure gauge diagnostic which is contained in PP21, too, but will not be discussed further in this paper.

The following sections give an overview of the results achieved by the combined effort of all partners in the ITER bolometer cluster during the past year.

\footnotetext{
a) Contributed paper, published as part of the Proceedings of the 17th Topical Conference on High-Temperature Plasma Diagnostics, Albuquerque, New Mexico, May 2008.

b)Electronic mail: meister@ipp.mpg.de.
}

\section{BOLOMETER DETECTOR DEVELOPMENT}

The plasma radiation over a wide spectral range (from soft- $X$ to the infrared) is captured in a bolometer within an absorber, which is thus heated. The increase in temperature is monitored using a thermometer giving thus the possibility to deduce the absorbed radiational power. Depending on the type of this thermometer, the different variants of bolometers are distinguished. Metal resistor bolometers use a thin metal resistor on the back side of the absorber ${ }^{1}$ combined with a reference detector, which is shielded from the direct plasma radiation to compensate for uncertainties in the measurement due to changes in temperature of the environment or the effect of neutron radiation. Imaging bolometers use a thin metal foil behind a pin hole whose temperature is monitored by an IR charge coupled device camera. ${ }^{2}$ Capacitive bolometers make use of the change in capacitance of a capacitor due to temperature changes induced by plasma radiation. ${ }^{3} \mathrm{An}$ assessment of the alternative detector types revealed that they are not yet as far advanced with respect to their application in operating fusion devices and their development for the use in ITER as the metal resistor bolometer. Therefore, the latter detector type was chosen as the reference detector type for ITER and will be the only detector type considered in this work. However, IR imaging and capacitive bolometers might well be considered as an upgrade or extension of the ITER bolometer diagnostic in the future.

Metal resistor bolometers in operating fusion devices use a gold absorber on thin mica or kapton foils with gold meanders on their back side. Owing to the high neutron flux expected in ITER, these bolometers would eventually fail because of the transmutation of $\mathrm{Au}$ to $\mathrm{Hg}$ and the embrittlement of the mica or kapton foils. Thus, first samples of metal resistor bolometers using a $\mathrm{Pt}$ absorber on a $\mathrm{SiN}$ foil have 
been produced and tested. ${ }^{4}$ The sputtering process used so far for the deposition of the absorber on the SiN foil allowed the production of absorbers of about $3.5 \mu \mathrm{m}$ thickness which exhibited an enhanced sensitivity and good long-term behavior in ASDEX Upgrade. However, the higher temperatures expected in ITER require Pt absorbers of at least $12 \mu \mathrm{m}$ thickness. Their development is a crucial part of the bolometer development for ITER.

Additionally, the meanders and their contacting by the signal cables have to be optimized for ITER. The contacting of the bolometer detectors requires reliably bonded contacts by Pt bonding. First tests were successful and resulted in tear-off forces of $11 \mathrm{~g}$ which is relatively high when compared to $2.5 \mathrm{~g}$ for Au bonding. The resistances of measurement and reference meander have to be matched exactly in order to prevent thermal drifts. Therefore, the method of laser trimming has been tested on first samples. The resistances of measurement and reference meander could be matched to within $1 \%$ accuracy at values of $\approx 1300 \Omega$. After optimization of the laser-trimming procedure and the layout of the meanders, accuracies of $0.1 \%$ are expected. Experience from measurements at WENDELSTEIN 7AS indicates a drift of the measured bridge imbalance of $100 \mu \mathrm{V} / \mathrm{K}$ at $2 \%$ resistance mismatch. Scaled to the $0.1 \%$ mismatch expected for ITER this would mean having a drift of $5 \mu \mathrm{V} / \mathrm{K}$ or, using the maximum increase in temperature of $150 \mathrm{~K}$ of the detector during a discharge in ITER standard scenario 2 (see sec. $\mathrm{V}$ ), the sensitivity of $4 \mathrm{~V} / \mathrm{W}$ of the amplifiers for ITER and the area of the sensors of $6 \times 10^{-6} \mathrm{~m}^{2}$, an increase in signal due to the change in temperature of the detector corresponding to a power deposition of about $30 \mathrm{~W} / \mathrm{m}^{2}$.

An improved data acquisition system for the bolometer diagnostic at ASDEX Upgrade has been taken into operation. This system features 32 channels per rack, a 16 bit ADC running at $1 \mathrm{kHz}$ sampling rate with an integration time of $10 \mathrm{~ms}$, and includes a calibration circuit which can be used for online calibration of the detectors in between discharges. ${ }^{5}$ Using a NI PXI 7833R FPGA, the LOS-integrated radiation power can be calculated in real time, enabling the diagnostic to send values for $P_{\text {tot }}$ and $P_{\text {div }}$ to the control system for radiation feedback. The minimum detectable signal at the amplifiers is $1 \mu \mathrm{V}$ ( $1 \mathrm{bit}$ ). Thus, the maximum detectable signal at the amplifiers is $65 \mathrm{mV}$, corresponding to a power density of $2.7 \mathrm{~kW} / \mathrm{m}^{2}$ at the detector foils. This system is directly transferable and in terms of number of channels also scalable to the requirements of ITER. Thus, the specifications as stated in this paragraph are used for calculating expected signal levels in ITER for the following sections.

\section{LINE-OF-SIGHT OPTIMIZATION}

Bolometers provide only a line-integrated measurement of the plasma radiation along lines of sight which are defined by the geometry of the detectors. A spatially resolved profile measurement is possible by means of many detectors with crossing lines of sight and the application of tomographical reconstruction methods. Figure 1 shows the distribution of all LOSs projected into one poloidal plane. This distribution is based on the results of an extensive optimization

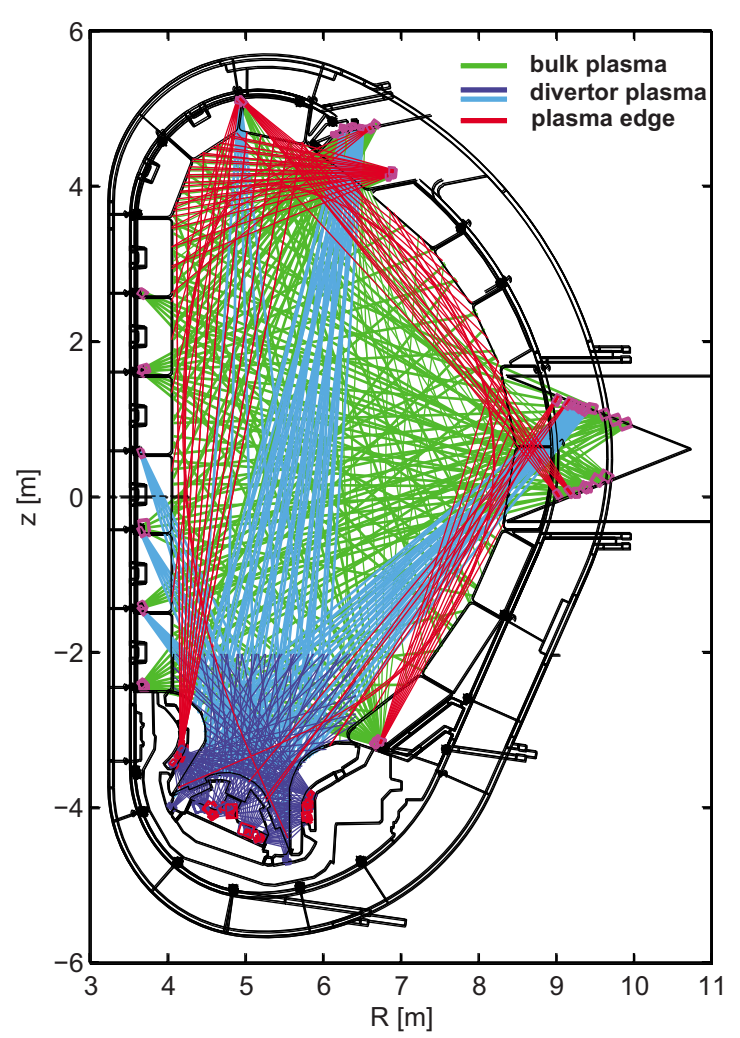

FIG. 1. (Color online) All LOSs for the ITER bolometer diagnostic projected into one poloidal plane.

procedure, ${ }^{6,7}$ while taking into account the technical constraints of the ITER vacuum vessel. It features an improved coverage of all plasma regions by covering critical areas individually. From the total number of 525 LOSs, organized in 105 minicameras with 5 LOSs each, 90 LOSs observe only the plasma edge (red in Fig. 1), 180 LOSs observe only the bulk plasma (green in Fig. 1), and 255 LOSs observe the divertor region, including the $X$-point region. In particular, the divertor plasma is observed directly from the divertor (dark blue in Fig. 1) and from outside the divertor region (light blue in Fig. 1) simultaneously in order to allow for the compensation of the charge-exchange neutrals which may contribute to the signal measured by the detectors in the divertor cassettes. In the toroidal direction most LOSs for the bulk plasma have been concentrated in port 1 (150 LOSs) to avoid uncertainties due to toroidal asymmetries in the radiation profile. In particular, for LOS observing the plasma edge and the divertor region, similar views in different toroidal locations allow for the quantitative measurement of toroidal asymmetries and provide a level of redundancy in the case of failures.

Additionally, it has been assessed that the views into the outer divertor from behind the inner heat shield could be replaced by toroidally viewing detectors in the port plugs. This alternative would reduce the risk of losing information due to the failing detectors as the ones behind the inner heat shield might not be replaceable during the whole lifetime of ITER. The required views and the same spatial resolution as from the detectors on the vacuum vessel wall can be achieved by dedicated minicameras in the upper port plugs. However, it is questionable if they can be implemented in 


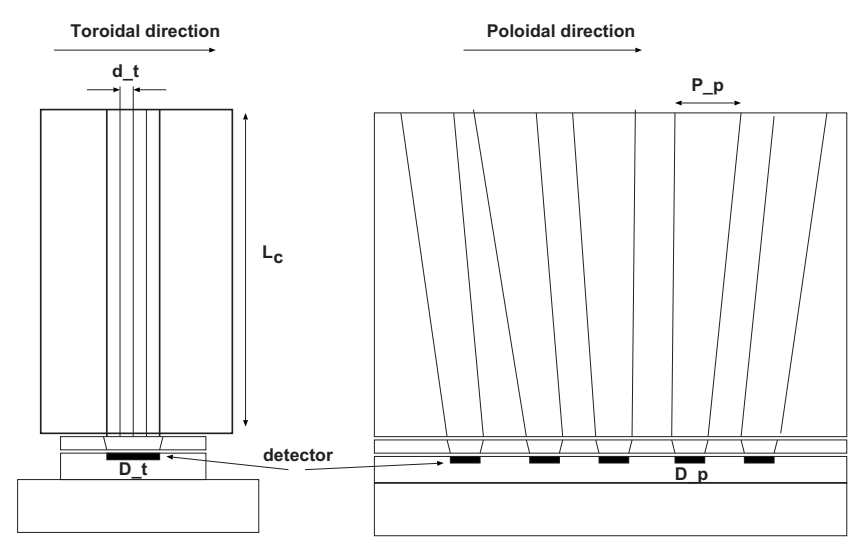

FIG. 2. Principal layout of the complex collimator.

ITER because they would require relatively large housings and their toroidally tilted viewing cones would most probably result in clashes with other diagnostics in the center of the port plugs.

A detailed analysis of the viewing geometry of the minicameras revealed that due to the small size of the gap between blanket modules, the collimators of all minicameras need to be divided into at least two subsections in the toroidal direction in order to prevent that the viewing cone is limited by the blanket modules. The principal layout of such a collimator is shown in Fig. 2. The realization of this kind of collimator will be a challenge to the engineers.

\section{PERFORMANCE ASSESSMENT}

The performance of the bolometer diagnostic has been assessed based on the parameters of the ITER standard scenario 2. Calculations have been made to assess the expected signal ranges, the affordable positioning inaccuracy, and the quality of the tomographic reconstruction. ${ }^{8}$

The expected signal ranges of all detectors have been calculated using the geometry resulting from the LOS optimization (Sec. III). The highest signals are expected for the detectors in the divertor cassettes. The high plasma radiation in this region results in signal levels in the order of $1 \mathrm{~kW} / \mathrm{m}^{2}$. The signal levels of the detectors observing the bulk radiation will be $\approx 20 \mathrm{~W} / \mathrm{m}^{2}$, and those for the detectors observing the plasma edge $\approx 6 \mathrm{~W} / \mathrm{m}^{2}$. The noise level in ASDEX Upgrade is about twice as high as the minimum electronic noise of 1 bit. To guess the noise level in ITER, we have to add a factor of 3 due to the thicker absorber and, to be very conservative, another factor of 4 to allow for additional noise due to the irradiation effects for which no experience exists so far. This would correspond to a power density at the detector of $1 \mathrm{~W} / \mathrm{m}^{2}$. This is $10 \%-20 \%$ of the value expected for the detectors observing the plasma edge and shows that the design of the collimators of these detectors needs a very careful optimization between achievable light yield and well-defined viewing cones. Comparing the expected signal levels of the various detectors with the signals due to thermal drifts (Sec. II), it becomes clear that for the detectors observing bulk and edge plasma an online correction of the thermal drifts is highly desirable. To prepare for this correction, the data acquisition system is equipped with the possibility of measuring the current through the detector Wheatstone bridge online, thus having a measurement of the temperature of the detector. Laboratory tests to relate this measurement to the thermal drift are in preparation.

A misalignment with respect to the ideal orientation of the LOS in poloidal direction will result in changed measurement values due to the LOS viewing different plasma regions than intended. This results in acceptable signal variations $(<0.5 \%)$ if the alignment does not differ more than $0.01 \mathrm{rad}$ from the ideal direction. However, a misalignment in the toroidal direction can have a stronger impact if it results in shadowed viewing cones due to target surfaces limiting the gap in front of the detectors. A quantitative assessment of the toroidal misalignment is planned for the near future.

The tomographic method used to reconstruct the radiation profile from sight-line integrated measurements is based on series expansion constraint optimization with regularization. A detailed description of this method is given in Ref. 6 and partly in Ref. 9. For quantitative comparisons of the original radiation profile and the one reconstructed using the expected measurement values with added random noise of various amplitudes $(1 \%-5 \%$ of the expected power density of the respective detector in ITER standard scenario 2), a number of scalar or one dimensional parameters have been derived from the two dimensional radiation profile. This includes, e.g., the total radiation $P_{\text {rad,tot }}$ in the whole plasma or only in specific regions such as bulk, divertor or $X$-point region, the position and amplitude of a ficticious $m=1$ perturbation in the radiation profile, and the position of the strike points. For high-quality measurements (1\%-2\% random noise), a good capability of reconstructing the radiation profile has been found. The achievable spatial resolution at the plasma edge in those regions covered by LOS is $\approx 10 \mathrm{~cm}$ (at $2 \%$ random noise). The ITER measurement requirements ${ }^{10}$ can be achieved with moderate noise levels of $2 \%-4 \%$. This conclusion remains valid also when taking the expected levels of power density at the detectors and noise in ITER into account, as the LOS observing the plasma edge are not necessary for the determination of the total plasma radiation. However, it is also obvious that measurements of the edge gradient of the plasma radiation in ITER will be successful only in case the noise levels are not as high as the conservative assumption made above.

\section{DRAFT DESIGN OF COMPONENTS}

The advances in the design of the components were driven by the needs of the ITER organization to define the interfaces for the divertor cassettes and for the vacuum vessel. For this purpose a generic design of a bolometer minicamera, a combination of five measurement and five reference detectors (i.e., five LOSs) in one housing with a collimator on top, has been produced. From this design the maximum dimensions of the minicamera have been defined: the detector housing will be $80 \times 90 \mathrm{~mm}^{2}$ and $45 \mathrm{~mm}$ high; the length of the collimator will be at most $130 \mathrm{~mm}$, varying strongly with the requirements of each individual location. The generic minicamera has been placed on all bolometer locations in the divertor cassettes while observing the re- 


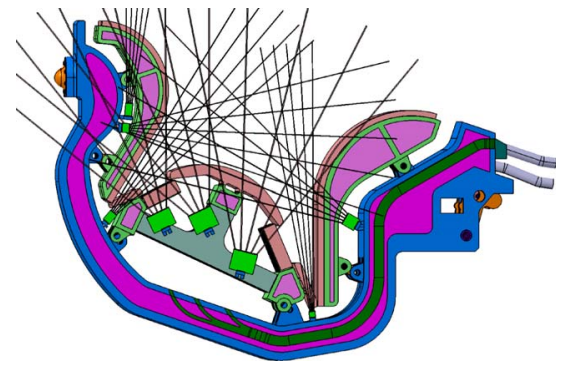

FIG. 3. (Color online) Eight bolometer minicameras placed in divertor cassette 21 (port 8).

quirements of the individual viewing geometries and adjusting the collimators, respectively. As an example, the placement of the minicameras in divertor cassette 21 (port 8) is shown in Fig. 3. The same exercise is about to be finished for the minicameras on the wall of the vacuum vessel which is being performed with the assistance of the ITER diagnostic group and by the ITER design office.

In order to assess if the minicameras will be able to withstand the operating conditions in ITER, thermal and EM loads finite-element analysis have to be carried out. Initially, thermal load calculations for three different minicameras in ITER have been performed for the conditions of standard scenario 2. Neutronics calculations have been performed by Monte Carlo MCNP5 radiation transport code ${ }^{11}$ in a three dimensional (3D) model of ITER, which was automatically generated from CAD models by means of the McCad interface program. ${ }^{12}$ The 3D MCNP model consists of a $40^{\circ}$ toroidal sector of ITER-FEAT with reflective boundary conditions on the lateral sides. The nuclear heating was normalized on $500 \mathrm{MW}$ fusion power. The nuclear heating results include neutron and gamma depositions. State-of-the-art FENDL-2.1 nuclear data library has been used for the neutronics analysis. The homogeneous material compositions have been assigned for the blanket and vacuum vessel surrounding the minicameras. CATIA models of the respective minicameras have been converted to the MCNP input deck using the McCad interface and placed on their intended locations for calculating the nuclear heating load. The highest thermal loads on the detector housing found are $0.23 \mathrm{~W} / \mathrm{cm}^{3}$ (value averaged over all MCNP cell volumes), and those on the detector foils are $0.1 \mathrm{~W} / \mathrm{cm}^{3}$ (both midplane on the HFS vacuum vessel). Of course, these vary strongly with location and conditions. In general, these values scale with the overall neutron flux at the wall in ITER as given in Figs. 4.1-5 of Ref. 13.

Using the calculated values of the nuclear thermal load and the projected thermal loads due to plasma radiation, the collimator of a typical minicamera on the inboard VV will heat up to $450{ }^{\circ} \mathrm{C}$ and the detector foil holder (heat sink for the foils) will heat up to about $300{ }^{\circ} \mathrm{C}$ (compared to the reference operating temperature of $150{ }^{\circ} \mathrm{C}$ ) in the case of a collimator made of SS316L and passive cooling. The maximum temperature can be reduced by using materials with enhanced thermal conductance with respect to stainless steel such as copper or TZM.

\section{THE ROADMAP TO FINAL ITER BOLOMETER DIAGNOSTIC}

One of the most crucial developments for the final ITER bolometer diagnostic is the development of a radiation hard resistive bolometer foil with thick enough absorbers to reliably detect the total radiated power from the plasma. The aim is to develop a resistive bolometer detector with Pt absorber of at least $12 \mu \mathrm{m}$ thickness on a $\mathrm{SiN}$ foil. Irradiation tests of the first samples (see Sec. II) are scheduled for October 2008. Irradiation tests with further more advanced samples of later development stages are planned. The detector development will be continued with a newly started industrial cooperation. The series production for ITER is expected for mid-2011. Additionally, the ongoing bolometer detector optimization will encompass the implementation of the laser trimming of meanders in order to match resistances of measurement and reference detector to within $0.1 \%$. Tests on prototypes of the foils are planned in order to prove their reliable operation under ITER conditions, including durability against high operating temperatures, EM-induced forces, and sudden pressure increase and the test of the envisaged earthing scheme.

From the engineering point of view, the generic design of the collimator which reliably defines the viewing geometry, inhibits the effects of stray light and maximizes the light yield is a challenge. Additionally, the housing of the detector and especially the collimator has to be designed with regard to damping any ECRH stray radiation which might deposit similar powers on the absorber as the plasma radiation and thus result in false measurements. A first design has been proposed $^{14}$ and has to be adapted to the complex collimator (Fig. 2). The demands on the collimator with respect to accuracy and ability to inhibit stray light and stray ECRH radiation require tests of prototypes. It is planned to prepare tests to check the capability of damping stray light and ECRH radiation and to check the manufacturing accuracy and resulting geometrical properties. While some errors in the poloidal alignment of the minicameras with respect to the design can be tolerated, the resulting orientation has to be known and thus measured to guarantee the calibration of the geometrical function of the diagnostic for a successful tomographic reconstruction of the radiation profile. To this aim the development of an in situ calibration of the LOS geometry is planned and will be tested in ASDEX Upgrade. The principle relies on the measurement of the detector signal in response to a bright light source which is moved along wellknown coordinates inside the vacuum vessel.

Additional engineering tasks are the development of structures for the supply of active cooling in the divertor cassettes and the performance of finite-element analysis. The latter will be used to check the thermal and EM loads on the minicameras which in turn are used to estimate operating temperatures, expected distortions, and forces on the housing and thus can be used to define the most suitable materials and designs for collimators and detector housings.

The integration of all diagnostic components in the locations envisaged for ITER will include to a large extent the resolving of clashes with structures and other diagnostics. Minor changes of the coordinates of the minicameras due to 
the technical constraints are expected. They will have to be used for improving the LOS optimization and to redo the performance analysis. The calculation of the expected signal ranges and error sources gives valuable input to the design of components by defining the maximum affordable tolerances in manufacturing minicameras. Lastly, the performance analysis on the final design has to show whether the ITER measurement requirements can be met.

\section{ACKNOWLEDGMENTS}

This work has been performed within the framework of a task of the EFDA Technology Workprogramme. The fruitful discussions with the EFDA responsible officer are warmly acknowledged. It is a pleasure to acknowledge the excellent support by the ITER diagnostic group.

${ }^{1}$ K. F. Mast, J. C. Vallet, C. Andelfinger, P. Betzler, H. Kraus, and G. Schramm, Rev. Sci. Instrum. 62, 744 (1991).

${ }^{2}$ B. J. Peterson, Rev. Sci. Instrum. 79, 10E301 (2008).

${ }^{3}$ V. Zauls, K. Kundzins, A. Sternbergs, I. Aulika, M. Kundzins, R. Bittner, K. Humer, and H. W. Weber, Contr. EFDA-IRRCER Meeting, Garching 6./7. April 2006.
${ }^{4}$ L. Giannone, D. Queen, F. Hellman, and J. C. Fuchs, Plasma Phys. Controlled Fusion 47, 2123 (2005).

${ }^{5}$ L. Giannone, K. F. Mast, M. Schubert, N. Team, E. Team, and W. Team, Rev. Sci. Instrum. 73, 3205 (2002).

${ }^{6}$ S. Kalvin, Tech. Rep., Final Report to EFDA Contract 03-1116, Association EURATOM-HAS, 2006.

${ }^{7}$ L. C. Ingesson, B. Alper, B. J. Peterson, and J.-C. Vallet, in Plasma Diagnostics for Magnetic Fusion Research, edited by A. E. Costley and D. W. Johnson, special issue on Fusion Sci. Technol. 53, 527-576 (2008).

${ }^{8}$ S. Kalvin and G. Grunda, Tech. Report, Final Report to EFDA Contract 06-1447 (TW6-TPDS-DIADES-5.2), Association EURATOM-HAS, 2008.

${ }^{9}$ S. Kálvin and L. C. Ingesson, Ann. Isr. Phys. Soc. 988, 485 (2008).

${ }^{10}$ J. How, Tech. Report., ITER Organisation, IDM: ITER D 2234RH, 2007.

${ }^{11}$ X-5 Monte Carlo Team, MCNP-A general Monte Carlo N-particle transport code, Version 5, Volume I: Overview and theory, Los Alamos National Laboratory, 24 April 2003, LA-UR-03-1987.

${ }^{12} \mathrm{H}$. Tsige-Tamirat, and U. Fischer, CAD interface for Monte Carlo particle transport codes, Proc., Monte Carlo 2005 Topical Meeting, The Monte Carlo Method: Versatility Unbounded in a Dynamic Computing World (Chattanooga, TN, 17-21 April 2005) on CD-ROM (LaGrange Park, IL: American Nuclear Society).

${ }^{13}$ H. Ida, V. Khripunov, L. Petrizzi, G. Federici, and N. A. Group, Tech. Report, ITER Organisation, IDM: ITER_D_22FST v2.0, ITER Naka \& Garching Joint Work Sites, 2004.

${ }^{14}$ R. Reichle and S. Hourcade, Tech. Report, Final Report to EFDA Contract 03-1111, 2007. 